\title{
Maternal deaths: Think of rare causes when common causes have been eliminated
}

\author{
Rathnaweera RHAI ${ }^{1}$, Vidanapathirana $\mathbf{M}^{2}$ \\ ${ }^{1}$ Lecturer, Department of Forensic Medicine, Faculty of Medicine, Galle, ${ }^{2}$ Senior Lecturer, Department of \\ Forensic Medicine, Faculty of Medical Sciences, University of Sri Jayewardenepura, Sri Lanka.
}

\author{
Correspondence: $\quad$ R.H.A.I. Rathnaweera \\ Department of Forensic Medicine, Faculty of Medicine, Galle, Sri Lanka. \\ e-mail: ajithrathnaweera@yahoo.com
}

\section{Introduction}

In Sri Lanka, maternal mortality ratio is among the lowest when compared with other developing counties $(1,2)$. Most deliveries take place in a health facility with the support of a skilled birth attendant. It is documented that during an hour, around 40 maternal deaths occur worldwide, whereas in Sri Lanka 40 women would die over a period of about 105 days (3). Once the common causes of maternal mortality have been controlled or eliminated, the uncommon causes come into picture.

Case 1: A 30-year-old pregnant mother with a period of amenorrhoea (POA) of 32 weeks was transferred from a peripheral hospital with vomiting and icterus for three days. She was suspected of having HELLP syndrome. Investigations showed abnormal liver, renal and clotting profiles. Emergency caesarian section was performed and after the delivery she developed profuse post-partum haemorrhage. Subtotal hysterectomy was done and she was transfused with blood, plasma and platelets. Her liver, renal functions and platelet count continued to deteriorate. A week after delivery she died in spite of ICU care.

At autopsy, yellow discolouration of conjunctiva, nail beds, pleural effusion and ascites was detected. All the organs were yellowish. Lungs were congested and heavy (Figure 1). Heart was flabby and Liver was enlarged. Kidneys were soft and enlarged with congested cortex. Histopathology revealed extensive pulmonary haemorrhages (Figure 2) and focal hepatic necrosis. Kidneys showed evidence of acute renal tubular necrosis.

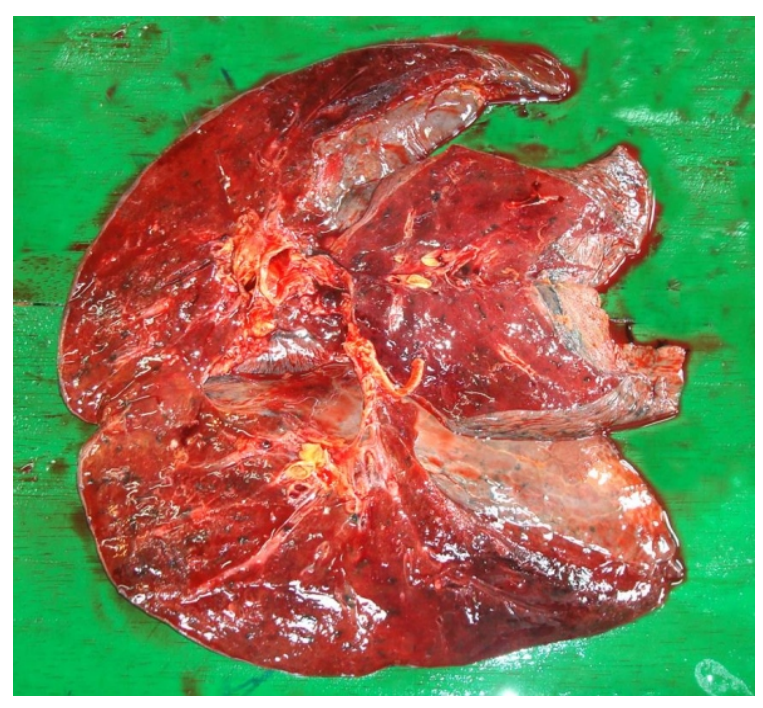

Figure 1: Congested lungs

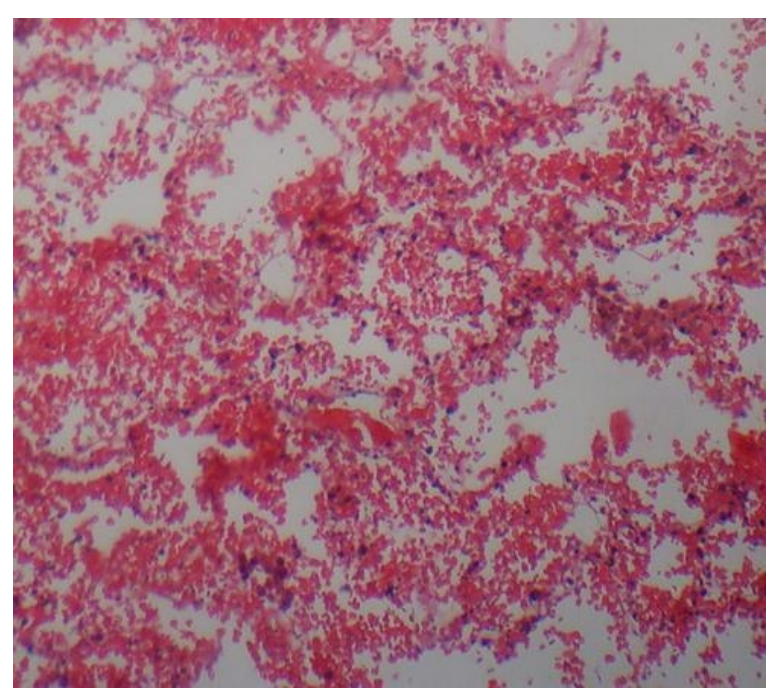

Figure 2: Pulmonary haemorrhages 
Though the clinicians treated this mother as complicated HELLP syndrome with multi-organ failure, the possibility of leptospirosis was not considered. Microscopic agglutination of leptospira antibody test was positive with a titer of 1600 . Cause of death was leptospirosis.

Although, HELLP syndrome is specific to pregnant state, leptospirosis is rarely described and it may mimic puerperal sepsis or hepatorenal failure (4). Though the most common causes of acute liver failure are viral hepatitis, drugs, and toxins, leptospirosis also can cause hepatitis and acute liver failure (5). Approximately $10 \%$ of those infected with leptospira develop jaundice with hepatocellular necrosis, and massive pulmonary haemorrhage (6). Early recognition and treatment would have prevented this death.

Case 2: A 23-year-old mother developed abdominal pain and convulsions two days after the delivery. Later she developed bile stained vomiting. Intestinal obstruction was suspected and emergency laparotomy was performed and necrotic bowel segments were removed. Her condition gradually deteriorated. At autopsy, faecal peritonitis and (Figure 3) infarctions in the spleen (Figure 4) were detected. Histopathology of the bowel revealed a preexisting vascular disease (vasculitis) with thickened vessel walls but the type of vasculitis could not be ascertained (Figure 5). Histopathology of the spleen also showed vasculitis with thickened walls.

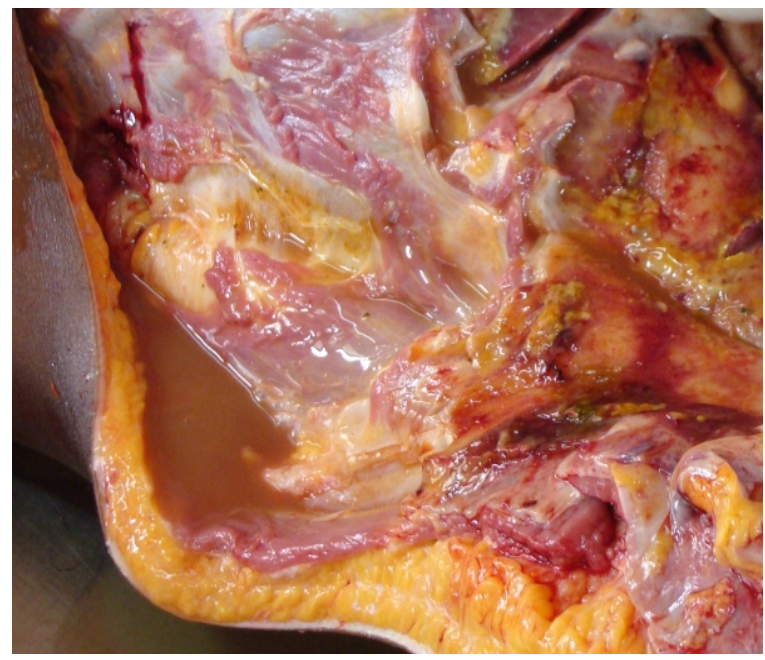

Figure 3: Faecal peritonitis

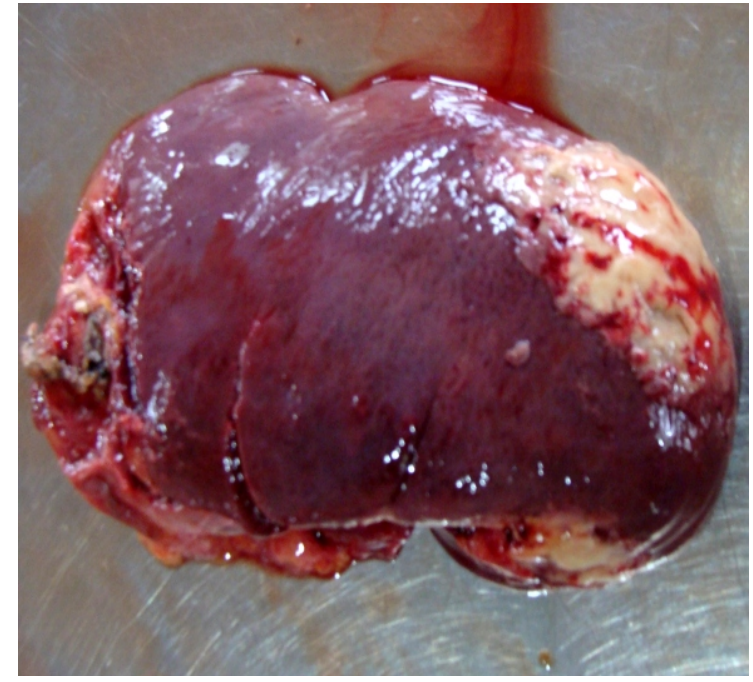

Figure 4: Infarctions in spleen

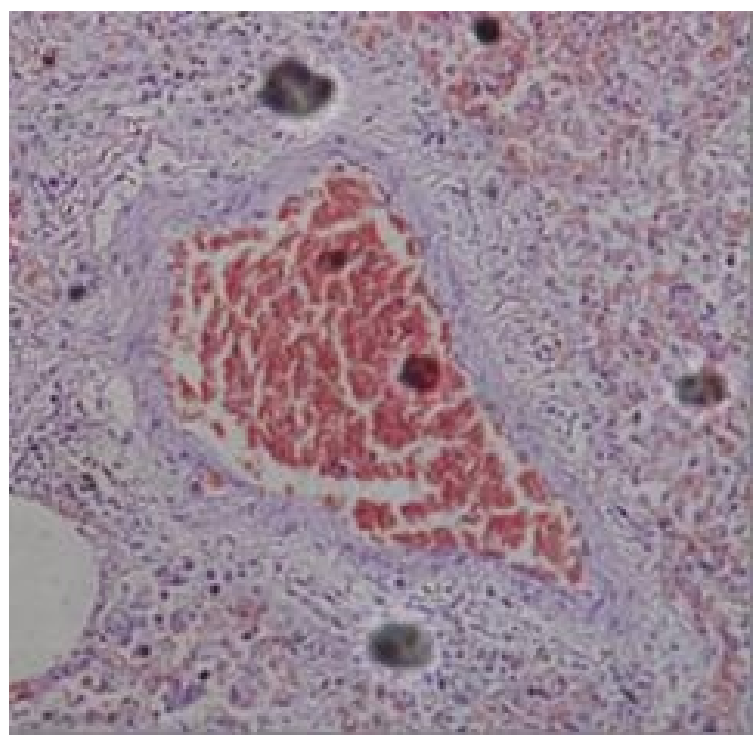

Figure 5: Vasculitis of bowels with thickened vessel walls

This patient had vasculitis and necrosis of the bowel resulting intestinal obstruction. Cause of deaths was septicemia due to generalized peritonitis following spontaneous bowel rupture due to vasculitis of the bowel. At the maternal mortality review, it was revealed that the patient had an episode of polyarthritis and defaulted treatment. Vasculitis can interrupt blood supply to tissues and organs causing tissue damage and even death. Splenic infarctions could be related to vasculitis (7). Some forms of vasculitis are self-limiting but others require treatment (8). In this case, the importance of obtaining a detailed history is highlighted. 
Case 3: A 29-year-old mother developed vomiting, fever, abdominal pain, icterus and altered level of consciousness one week after delivery. She was admitted to hospital and managed as HELLP syndrome. In spite of treatment, her condition deteriorated. At autopsy, dissection of the ears showed evidence of chronic otitis media. Brain was congested with purulent discharge in subarachnoid space. Histopathology revealed acute pyogenic meningitis.

Altered level of consciousness was suspected as a complication of HELLP syndrome with multi-organ failure though the cause of death was pyogenic meningitis. Otitis media is a well recognized cause of pyogenic meningitis (9).

In conclusion, detailed history taking, meticulous investigation and multi-disciplinary analysis are important to prevent maternal deaths due to rare causes during post-partum period.

\section{References}

1. World Health Organization, UNICEF, UNFPA and World Bank 2005. Maternal Mortality, estimates developed by World Health Organization.

2. Medical Statistics Unit 2005. Annual Health Statistics Sri Lanka 2005. Ministry of Healthcare and Nutrition, Sri Lanka.
3. Attygalle D, Tillekaratne A, Niroshan I. 2008. Overview of Maternal Mortality in Sri Lanka 2001- 2005. Family Health Bureau, Colombo.

4. Hicham S, Ihsane M, Abderahim el B, Brahim B, Labib S, Mustapha H, Mohamed K, Adil I, Abdelilah M, Nabil K. Multivisceral organ failure related to leptospirosis in pregnant patient. Indian J Crit Care Med 2013; 17(1): 43-5.

5. Urganci NI, Kalyoncu D, Cayonu N, Erdem E, Yildirmak Y, Yilmaz B. Acute liver failure, autoimmune hepatitis, and leptospirosis: a case report. Pediatr Emerg Care 2011; 27(10): 963-5.

6. Leblebicioglu H, Sencan I, Sunbul M, et al. Weil's disease: report of 12 cases. Scand J Infect Dis 1996; 28(6): 637-9.

7. Rentsch J, McColl G. "Splenic infarction in Wegener's granulomatosis." J. Rheumatol 2000; 27 (6): 1554-5.

8. Koopman WJ, Boulware DW, Heudebert GR. Clinical Primer of Rheumatology, Lippincott Williams \& Wilkens, edited by William Koopman, et al, 2003.

9. Adriani KS, Brouwer MC, van der Ende A, van de Beek D. Bacterial meningitis in pregnancy: report of six cases and review of the literature. Clin Microbiol Infect 2012; 18(4): 345-51. 